\title{
Three Important Phenomena of Chaos Synchronization Between Two Different Hyperchaotic Systems via Adaptive Control Method
}

\author{
Maysoon M. Aziz, Saad Fawzi Al-Azzawi* \\ Department of Mathematics, College of Computer Sciences and Mathematics, University of Mosul, Mosul, Iraq \\ Email address: \\ saad_fawzi78@yahoo.com (S. F.Al-Azzawi) \\ ${ }^{*}$ Corresponding author
}

To cite this article:

Maysoon M. Aziz, Saad Fawzi Al-Azzawi. Three Important Phenomena of Chaos Synchronization Between Two Different Hyperchaotic Systems via Adaptive Control Method. International Journal of Theoretical and Applied Mathematics. Vol. 3, No. 1, 2017 , pp. 36-42. doi: $10.11648 /$ j.ijtam.20170301.16

Received: September 26, 2016; Accepted: December 10, 2016; Published: January 12, 2017

\begin{abstract}
This paper presents three important phenomena of chaos synchronization between two different hyperchaotic systems using nonlinear adaptive control strategy. In detailed, complete synchronization, anti- synchronization and hybrid synchronization with nine unknown parameters. Modified hyperchaotic Pan system is consider as drive and hyperchaotic Liu system as response system. Stabilization of error dynamics for each phenomenon is realized by satisfying Lyapunov's second method as a main tool. Theoretical analysis and numerical simulations are shown to verify the results.
\end{abstract}

Keywords: Complete Synchronization, Anti-synchronization, Hybrid Synchronization, Nonlinear Dynamical Systems, Adaptive Control, Lyapunov's Second Method

\section{Introduction}

Since the pioneering work of Pecora and Carroll in 1990s, on the synchronization of chaotic systems, synchronization phenomenon has formed a new body of research activities which is at the fore front of recent application topic in nonlinear dynamics [1-6], and opens the way for chaotic systems synchronization and a various techniques such as adaptive control, active control, nonlinear control, sliding mod control, back-stepping design method and so on have been successfully applied to chaos control and synchronization [7-13].

Various types of synchronization phenomena have been presented such as complete synchronization (CS), generalized synchronization (GS), lag synchronization, antisynchronization (AS), projective synchronization (PS), generalized projective synchronization (GPS). The most familiar synchronization phenomena are complete synchronization and anti- synchronized [14], [15].

Complete synchronization is characterized by the equality of state variables evolving in the time, while antisynchronization is characterized by the disappearance of sum of relevant variables evolving in the time. Projective synchronization is characterized by the fact that the drive and response systems could be synchronized up to a scaling factor, whereas in generalized projective synchronization, the responses of the synchronized dynamical states synchronize up to a constant scaling matrix $\alpha$. It is easy to see that the complete synchronization and antisynchronization are special cases of generalized projective synchronization where the scaling matrix $\alpha=I$ and $=-I$, respectively [16].

In the hybrid synchronization scheme, one part of the system is synchronized and the other part is antisynchronized so that the complete synchronization and antisynchronization coexist in the system [17].

In this paper, we discuss some important phenomena for chaos synchronization i.e. complete synchronization, antisynchronization and hybrid synchronization between two different 4D hyperchaotic systems via adaptive control, The results derived in this paper are established using the Lyapunov's second method. 


\section{Description of Hyperchaotic Modified Pan and Liu Systems}

According to the Ref [18], the modified hyperchaotic Pan system which described by the following dynamical system

$$
\left\{\begin{array}{c}
\dot{x}_{1}=a\left(x_{2}-x_{1}\right) \\
\dot{x}_{2}=c x_{1}-x_{1} x_{3}+x_{4} \\
\dot{x}_{3}=x_{1} x_{2}-b x_{3} \\
\dot{x}_{4}=-d x_{2}
\end{array}\right.
$$

where $x_{1}, x_{2}, x_{3}, x_{4}$ are the state variables and $a, b, c, d>0$ are the parameters of the system. When parameters $a=$ $10, b=8 / 3, c=28$ and $d=10$, system (1) is hyperchaotic and has two positive Lyapunov exponents, i.e. $L E_{1}=$ $0.24784, L E_{2}=0.08194$, and has only one equilibrium
$O(0,0,0,0)$. This equilibrium is an unstable under these parameters.

In 2006, Wang et al. [19], presented the four-dimensional autonomous Liu system which described by

$$
\left\{\begin{array}{c}
\dot{x}_{1}=a\left(x_{2}-x_{1}\right) \\
\dot{x}_{2}=r x_{1}-k x_{1} x_{3}+x_{4} \\
\dot{x}_{3}=h x_{1}^{2}-p x_{3} \\
\dot{x}_{4}=-q x_{1}
\end{array}\right.
$$

where $a, r, k, h, p, q>0$ are system parameters. When parameters $\mathrm{a}=10, \mathrm{p}=2.5, \mathrm{r}=40, \mathrm{q}=10.6, \mathrm{k}=1 \quad$ and $\mathrm{h}=4$, system (2) is hyperchaotic and has only equilibrium $\mathrm{O}(0,0,0,0)$, and the equilibrium is an unstable saddle node under these parameters. Fig. 1 and Fig. 2 shows the attractors of the system (1) and the system (2) respectively.

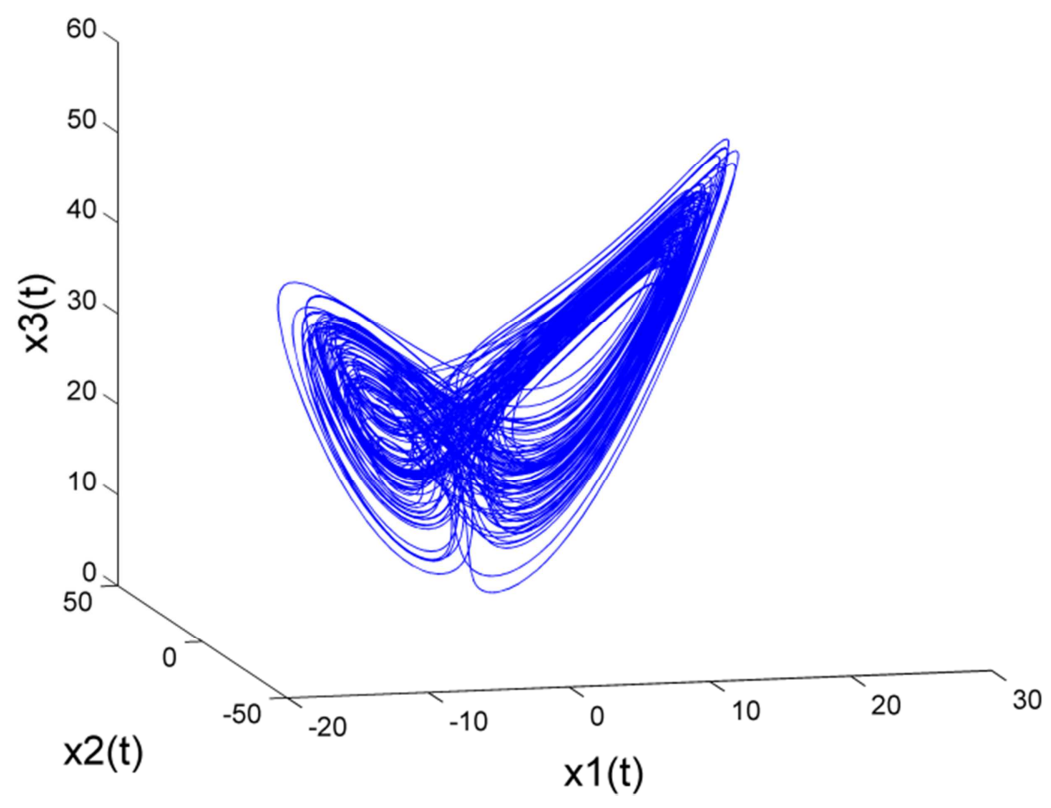

Fig. 1. The attractor of system (1) in $x_{1}, x_{2}, x_{3}$ space.

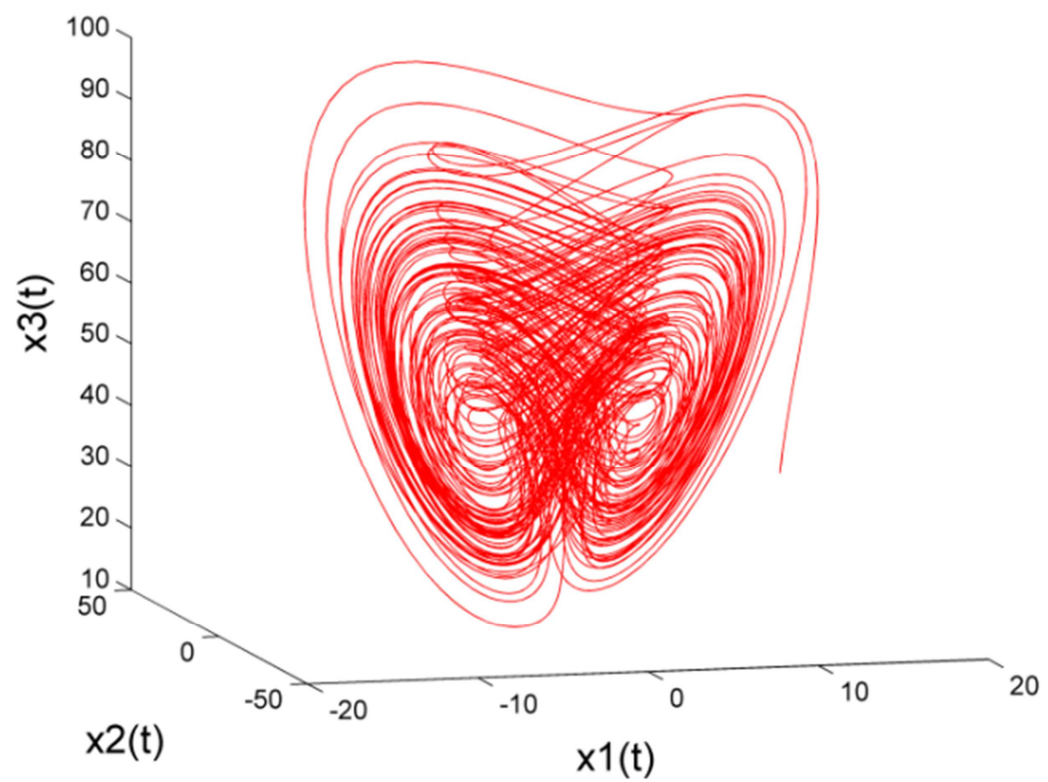

Fig. 2. The attractor of system (2) in in $x_{1}, x_{2}, x_{3}$ space. 


\section{Complete Synchronization Between Modified Pan and Liu Systems}

In this section, the synchronization behavior between two different hyperchaotic systems with nine unknown parameters is achieved based on the Lyapunov second method and adaptive control strategy. In order to observe complete synchronization between hyperchaotic Modified Pan system and hyperchaotic Liu system, we consider the system (1) as the drive system and hyperchaotic Liu system as the response system which describe by the following system

$$
\left\{\begin{array}{c}
\dot{y}_{1}=a\left(y_{2}-y_{1}\right)+u_{1} \\
\dot{y}_{2}=r y_{1}-k y_{1} y_{3}+y_{4}+u_{2} \\
\dot{y}_{3}=h y_{1}^{2}-p y_{3}+u_{3} \\
\dot{y}_{4}=-q y_{1}+u_{4}
\end{array}\right.
$$

where $u=\left[u_{1}, u_{2}, u_{3}, u_{4}\right]^{T}$ is the controller to be designed.

The synchronization error $e \epsilon R^{4}$ is defined as:

$$
e_{i}=y_{i}-\alpha_{i} x_{i} ; i=1,2,3,4, \forall \alpha_{i}=1
$$

where $\alpha_{i}$ is a scaling factor taken the value 1 for complete synchronization and -1 for anti-synchronization according to the projective synchronization approach. So, subtracting the above system from the system (1), we get the error dynamical system between the drive system and the response system which is given by:

$$
\left\{\begin{array}{c}
\dot{e}_{1}=a\left(e_{2}-e_{1}\right)+u_{1} \\
\dot{e}_{2}=r e_{1}+e_{4}+(r-c) x_{1}-k y_{1} y_{3}+x_{1} x_{3}+u_{2} \\
\dot{e}_{3}=-p e_{3}+(b-p) x_{3}+h y_{1}^{2}-x_{1} x_{2}+u_{3} \\
\dot{e}_{4}=-q e_{1}-d e_{2}-q x_{1}+d y_{2}+u_{4}
\end{array}\right.
$$

We need to find the nonlinear adaptive control law for $u_{i}, \forall i$ and parameters estimation rules to guarantee that the error dynamics of (5) is globally asymptotically stable.

Choosing the control functions $u=\left[u_{1}, u_{2}, u_{3}, u_{4}\right]^{T}$ as

$$
\left\{\begin{array}{c}
u_{1}=(\hat{a}-1) e_{1}-\hat{a} e_{2} \\
u_{2}=-\hat{r} e_{1}-e_{2}-e_{4}+(\hat{c}-\hat{r}) x_{1}+\hat{k} y_{1} y_{3}-x_{1} x_{3} \\
u_{3}=(\hat{p}-1) e_{3}+(\hat{p}-\hat{b}) x_{3}-\hat{h} y_{1}^{2}+x_{1} x_{2} \\
u_{4}=\hat{q} e_{1}+\hat{d} e_{2}-e_{4}+\hat{q} x_{1}-\hat{d} y_{2}
\end{array}\right.
$$

where $\hat{a}, \hat{b}, \hat{c}, \hat{d}, \hat{h}, \hat{k}, \hat{p}, \hat{q}$ and $\hat{r}$ are estimates of the parameters $a, b, c, d, h, k, p, q$ and $r$, respectively.

Substituting the control law (6) into Eq.(5) gives

$$
\left\{\begin{aligned}
\dot{e}_{1}= & ((\hat{a}-a)-1) e_{1}-(\hat{a}-a) e_{2} \\
\dot{e}_{2}= & -(\hat{r}-r) e_{1}-e_{2}+((\hat{c}-c)-(\hat{r}-r)) x_{1} \\
& +(\hat{k}-k) y_{1} y_{3} \\
\dot{e}_{3}= & ((\hat{p}-p)-1) e_{3}+((\hat{p}-p)-(\hat{b}-b)) x_{3} \\
& -(\hat{h}-h) y_{1}^{2} \\
\dot{e}_{4}= & (\hat{q}-q) e_{1}+(\hat{d}-d) e_{2}-e_{4}+(\hat{q}-q) x_{1} \\
& -(\hat{d}-d) y_{2}
\end{aligned}\right.
$$

The parameter estimation errors are defined as

$$
\left\{\begin{array}{c}
\tilde{a}=\hat{a}-a ; \tilde{h}=\hat{h}-h \\
\tilde{b}=\hat{b}-b ; \tilde{k}=\hat{k}-k \\
\tilde{c}=\hat{c}-c ; \tilde{p}=\hat{p}-p \\
\tilde{d}=\hat{d}-d ; \tilde{q}=\hat{q}-q \\
\tilde{r}=\hat{r}-r
\end{array}\right.
$$

Substituting (8) into (7), the error dynamics simplifies to

$$
\left\{\begin{array}{c}
\dot{e}_{1}=\tilde{a}\left(e_{1}-e_{2}\right)-e_{1} \\
\dot{e}_{2}=-\tilde{r} e_{1}-e_{2}+(\tilde{c}-\tilde{r}) x_{1}+\tilde{k} y_{1} y_{3} \\
\dot{e}_{3}=(\tilde{p}-1) e_{3}+(\tilde{p}-\tilde{b}) x_{3}-\tilde{h} y_{1}^{2} \\
\dot{e}_{4}=\tilde{q} e_{1}+\tilde{d} e_{2}-e_{4}+\tilde{q} x_{1}-\tilde{d} y_{2}
\end{array}\right.
$$

As we known, the adaptive control strategy deals with unknown parameters only and used Lyapunov second method as a main tool. So, we need to construct a quadratic Lyapunov function as

$$
\begin{gathered}
V=\frac{1}{2}\left(e^{T} e+\tilde{a}^{2}+\tilde{b}^{2}+\tilde{c}^{2}+\tilde{d}^{2}+\tilde{h}^{2}+\tilde{k}^{2}\right. \\
\left.+\tilde{p}^{2}+\tilde{q}^{2}+\tilde{r}^{2}\right)
\end{gathered}
$$

The derivative of the Lyapunov function $V$ with respect to time is

$$
\begin{gathered}
\dot{V}=e_{1} \dot{e}_{1}+e_{2} \dot{e}_{2}+e_{3} \dot{e}_{3}+e_{4} \dot{e}_{4}+\tilde{a} \dot{\tilde{a}}+\tilde{b} \dot{\tilde{b}}+\tilde{c} \dot{\tilde{c}}+\tilde{d} \dot{\tilde{d}}+\tilde{h} \dot{\tilde{h}} \\
+\tilde{k} \dot{\tilde{k}}+\tilde{p} \dot{\tilde{p}}+\tilde{q} \dot{\tilde{q}}+\tilde{r} \dot{\tilde{r}}
\end{gathered}
$$

Also note that

$$
\left\{\begin{array}{cc}
\dot{\tilde{a}}=\dot{\hat{a}} ; & \dot{\tilde{h}}=\dot{\hat{h}} \\
\dot{\tilde{b}}=\dot{\hat{b}} ; & \dot{\tilde{k}}=\dot{\hat{k}} \\
\dot{\tilde{c}}=\dot{\hat{c}} ; & \dot{\tilde{p}}=\dot{\hat{p}} \\
\dot{\tilde{d}}=\dot{\hat{d} ;} & \dot{\tilde{q}}=\dot{\hat{q}} \\
\dot{\tilde{r}}= & \dot{\hat{r}}
\end{array}\right.
$$

$$
\begin{array}{r}
\dot{V}=e_{1}\left[\tilde{a}\left(e_{1}-e_{2}\right)-e_{1}\right]+e_{2}\left[-\tilde{r} e_{1}-e_{2}+(\tilde{c}-\tilde{r}) x_{1}+\tilde{k} y_{1} y_{3}\right]+e_{3}\left[(\tilde{p}-1) e_{3}+(\tilde{p}-\tilde{b}) x_{3}-\tilde{h} y_{1}^{2}\right]+e_{4}\left[\tilde{q} e_{1}+\tilde{d} e_{2}-\right. \\
\left.e_{4}+\tilde{q} x_{1}-\tilde{d} y_{2}\right]+\tilde{a} \dot{\hat{a}}+\tilde{b} \hat{\hat{b}}+\tilde{c} \dot{\hat{c}}+\tilde{d} \dot{\hat{d}}+\tilde{h} \hat{\hat{h}}+\tilde{k} \dot{\hat{k}}+\tilde{p} \dot{\hat{p}}+\tilde{q} \dot{\hat{q}}+\tilde{r} \dot{\hat{r}}
\end{array}
$$

According to the Lyapunov second method, to ensure the error dynamical system (9) converges to the origin

asymptotically, the condition $\dot{V}$ is negative i.e. $\dot{V}<0$. So we updated the estimated parameters by the following law: 


$$
\left\{\begin{array}{c}
\dot{\hat{a}}=-e_{1}^{2}+e_{1} e_{2}-\tilde{a} \\
\dot{\hat{b}}=x_{3} e_{3}-\tilde{b} \\
\dot{\hat{c}}=-x_{1} e_{2}-\tilde{c} \\
\dot{\hat{d}}=-e_{2} e_{4}+y_{2} e_{4}-\tilde{d} \\
\dot{\hat{h}}=y_{1}^{2} e_{3}-\tilde{h} \\
\dot{\hat{k}}=-y_{1} y_{3} e_{2}-\tilde{k} \\
\dot{\hat{p}}=-e_{3}^{2}-x_{3} e_{3}-\tilde{p} \\
\dot{\hat{q}}=-e_{1} e_{4}-x_{1} e_{4}-\tilde{q} \\
\dot{\hat{r}}=e_{1} e_{2}+x_{1} e_{2}-\tilde{r}
\end{array}\right.
$$

By this update and control (6), the time derivation of Lyapunov function $V$ becomes

$$
\begin{gathered}
\dot{V}=-e_{1}^{2}-e_{2}^{2}-e_{3}^{2}-e_{4}^{2}-\tilde{a}^{2}-\tilde{b}^{2}-\tilde{c}^{2}-\tilde{d}^{2}-\tilde{h}^{2}- \\
\tilde{k}^{2}-\tilde{p}^{2}-\tilde{q}^{2}-\tilde{r}^{2}<0
\end{gathered}
$$

Therefore, complete synchronization between two hyperchaotic systems (1) and (3) with nine unknown parameters is achieved.

Hence, we arrive at the following results.

Theorem 1. The non-identical system (1) and system (3) with nine unknown parameters are complete synchronize by nonlinear adaptive control law (6), and the update law for parameters is chosen as (13).

Numerically, we justified these analytical results by MATLAB program, we choose the parameters $a=10$, $b=8 / 3, c=28, d=10, \mathrm{p}=2.5, \mathrm{r}=40, \mathrm{q}=10.6, \mathrm{k}=$ 1 and $\mathrm{h}=4$ to ensure the system (1) and system (3) are hyperchaotic without control. Runge-Kutta method of four order is used with the initial values of the drive system and the response system are $(10,15,20,30)$ and $(-10,-5,0,5)$ respectively and the initial condition of the parameter update law as $\hat{a}(0)=3, \hat{b}(0)=7, \hat{c}(0)=-6, \hat{d}(0)=15$, $\hat{h}(0)=-4, \hat{k}(0)=12, \hat{p}(0)=-5, \hat{q}(0)=10, \hat{r}(0)=$ -2 . Fig. 3 and Fig. 4 show the complete synchronization between systems with adaptive control (6) and update parameters (13)
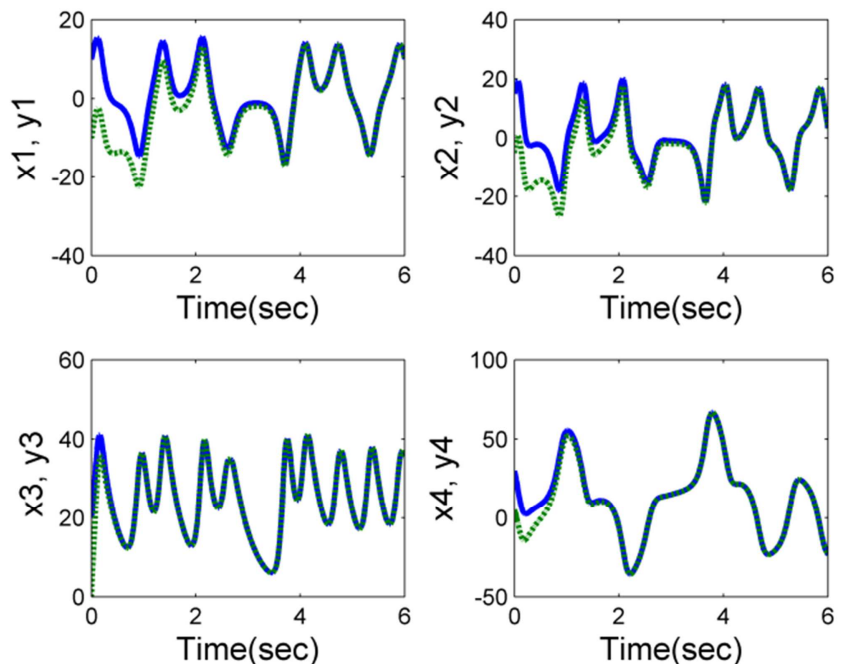

Fig. 3. Complete synchronization between systems (1) and (3) adaptive control (6) and update parameter (13).

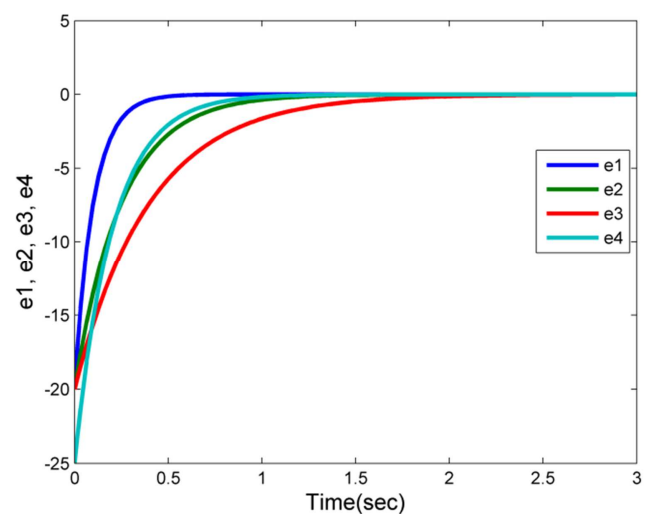

Fig. 4. The converges of system (5) with controllers (6) and update parameter (13).

\section{Anti-synchronization Between Modified Pan and Liu Systems}

In this section, we discuss anti-synchronization behavior of non-identical Modified hyperchaotic Pan system and hyperchaotic Liu system. Consider Modified hyperchaotic Pan as drive system and hyperchaotic Liu system as a response system (system3). We define the error states for anti- synchronization as

$$
e_{i}=y_{i}-\alpha_{i} x_{i} ; i=1,2,3,4, \quad \forall \alpha_{i}=-1
$$

The corresponding error dynamics system obtained by adding system(1) and system (3) is

$$
\left\{\begin{array}{l}
\dot{e}_{1}=a\left(e_{2}-e_{1}\right)+u_{1} \\
\dot{e}_{2}=r e_{1}+e_{4}+(c-r) x_{1}-k y_{1} y_{3}-x_{1} x_{3}+u_{2} \\
\dot{e}_{3}=-p e_{3}+(p-b) x_{3}+h y_{1}^{2}+x_{1} x_{2}+u_{3} \\
\dot{e}_{4}=-q e_{1}-d e_{2}+q x_{1}+d y_{2}+u_{4}
\end{array}\right.
$$

Theorem 2. System (1) will anti-synchronize with system (3) with nine unknown parameters if we choose the following adaptive controllers

$$
\left\{\begin{array}{c}
u_{1}=(\hat{a}-1) e_{1}-\hat{a} e_{2} \\
u_{2}=-\hat{r} e_{1}-e_{2}-e_{4}+(\hat{r}-\hat{c}) x_{1}+\hat{k} y_{1} y_{3}+x_{1} x_{3} \\
u_{3}=(\hat{p}-1) e_{3}+(\hat{b}-\hat{p}) x_{3}-\hat{h} y_{1}^{2}-x_{1} x_{2} \\
u_{4}=\hat{q} e_{1}+\hat{d} e_{2}-e_{4}-\hat{q} x_{1}-\hat{d} y_{2}
\end{array}\right.
$$

and the parameter estimation update laws

$$
\left\{\begin{array}{c}
\dot{\hat{a}}=-e_{1}^{2}+e_{1} e_{2}-\tilde{a} \\
\dot{\hat{b}}=-x_{3} e_{3}-\tilde{b} \\
\dot{\hat{c}}=x_{1} e_{2}-\tilde{c} \\
\dot{\hat{d}}=-e_{2} e_{4}+y_{2} e_{4}-\tilde{d} \\
\dot{\hat{h}}=y_{1}^{2} e_{3}-\tilde{h} \\
\dot{\hat{k}}=-y_{1} y_{3} e_{2}-\tilde{k} \\
\dot{\hat{p}}=-e_{3}^{2}+x_{3} e_{3}-\tilde{p} \\
\dot{\hat{q}}=-e_{1} e_{4}+x_{1} e_{4}-\tilde{q} \\
\dot{\hat{r}}=e_{1} e_{2}-x_{1} e_{2}-\tilde{r}
\end{array}\right.
$$


Proof. Applying control law (17) with (8) in system (16) yields the resulting error dynamics as follows:

$$
\left\{\begin{array}{c}
\dot{e}_{1}=\tilde{a}\left(e_{1}-e_{2}\right)-e_{1} \\
\dot{e}_{2}=-\tilde{r} e_{1}-e_{2}+(\tilde{r}-\tilde{c}) x_{1}+\tilde{k} y_{1} y_{3} \\
\dot{e}_{3}=(\tilde{p}-1) e_{3}+(\tilde{b}-\tilde{p}) x_{3}-\tilde{h} y_{1}^{2} \\
\dot{e}_{4}=\tilde{q} e_{1}+\tilde{d} e_{2}-e_{4}-\tilde{q} x_{1}-\tilde{d} y_{2}
\end{array}\right.
$$

According to the previous discussion, consider the Lyapunov function as

$$
\begin{gathered}
V=\frac{1}{2}\left(e_{1}^{2}+e_{2}^{2}+e_{3}^{2}+e_{4}^{2}+\tilde{a}^{2}+\tilde{b}^{2}+\tilde{c}^{2}+\tilde{d}^{2}+\tilde{h}^{2}+\tilde{k}^{2}+\tilde{p}^{2}+\tilde{q}^{2}+\tilde{r}^{2}\right) \\
\dot{V}=e_{1} \dot{e}_{1}+e_{2} \dot{e}_{2}+e_{3} \dot{e}_{3}+e_{4} \dot{e}_{4}+\tilde{a} \dot{\tilde{a}}+\tilde{b} \dot{\tilde{b}}+\tilde{c} \dot{\tilde{c}}+\tilde{d} \dot{\tilde{d}}+\tilde{h} \dot{\tilde{h}}+\tilde{k} \dot{\tilde{k}}+\tilde{p} \dot{\tilde{p}}+\tilde{q} \dot{\tilde{q}}+\tilde{r} \dot{\tilde{r}} \\
\dot{V}=e_{1}\left[\tilde{a}\left(e_{1}-e_{2}\right)-e_{1}\right]+e_{2}\left[-\tilde{r} e_{1}-e_{2}+(\tilde{r}-\tilde{c}) x_{1}+\tilde{k} y_{1} y_{3}\right] \\
+e_{3}\left[(\tilde{p}-1) e_{3}+(\tilde{b}-\tilde{p}) x_{3}-\tilde{h} y_{1}^{2}\right]+e_{4}\left[\tilde{q} e_{1}+\tilde{d} e_{2}-e_{4}-\tilde{q} x_{1}-\tilde{d} y_{2}\right] \\
+\tilde{a}\left[-e_{1}^{2}+e_{1} e_{2}-\tilde{a}\right]+\tilde{b}\left[-x_{3} e_{3}-\tilde{b}\right]+\tilde{c}\left[x_{1} e_{2}-\tilde{c}\right]+\tilde{d}\left[-e_{2} e_{4}+y_{2} e_{4}-\tilde{d}\right] \\
+\tilde{h}\left[y_{1}^{2} e_{3}-\tilde{h}\right]+\tilde{k}\left[-y_{1} y_{3} e_{2}-\tilde{k}\right]+\tilde{p}\left[-e_{3}^{2}+x_{3} e_{3}-\tilde{p}\right]+\tilde{q}\left[-e_{1} e_{4}+x_{1} e_{4}-\tilde{q}\right]+\tilde{r}\left[e_{1} e_{2}-x_{1} e_{2}-\tilde{r}\right] \\
\dot{V}=-e_{1}^{2}-e_{2}^{2}-e_{3}^{2}-e_{4}^{2}-\tilde{a}^{2}-\tilde{b}^{2}-\tilde{c}^{2}-\tilde{d}^{2}-\tilde{h}^{2}-\tilde{k}^{2}-\tilde{p}^{2}-\tilde{q}^{2}-\tilde{r}^{2}<0
\end{gathered}
$$

Therefore, anti-synchronization between two hyperchaotic systems (1) and (3) with nine unknown parameters is achieved. The proof is now complete based on Lyapunov method.

Numerically, we choose the parameters $a=10, b=$ $8 / 3, c=28, d=10, \mathrm{p}=2.5, \mathrm{r}=40, \mathrm{q}=10.6, \mathrm{k}=1 \quad$ and $\mathrm{h}=4$ the initial values of the drive system and the response system are $(10,15,20,30)$ and $(-10,-5,0,5)$ respectively and the initial condition of the parameter update law as $\hat{a}(0)=3, \hat{b}(0)=7, \hat{c}(0)=-6, \hat{d}(0)=15, \hat{h}(0)=$ $-4, \hat{k}(0)=12, \hat{p}(0)=-5, \hat{q}(0)=10, \hat{r}(0)=-2$. Fig. 5 and Fig. 6 show verify these results numerically.
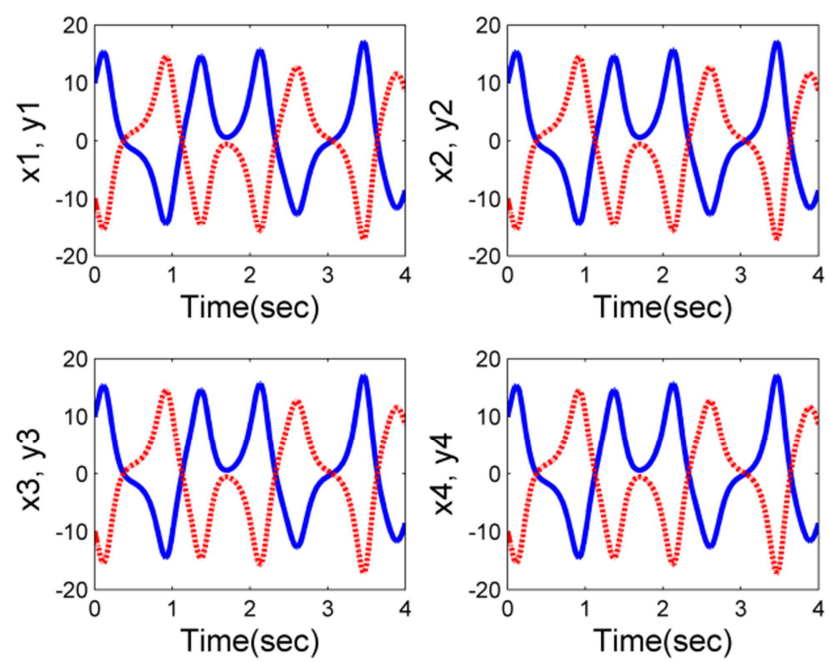

Fig. 5. Anti-synchronization between systems (1) and (3) with adaptive control (17) and update parameter (18).

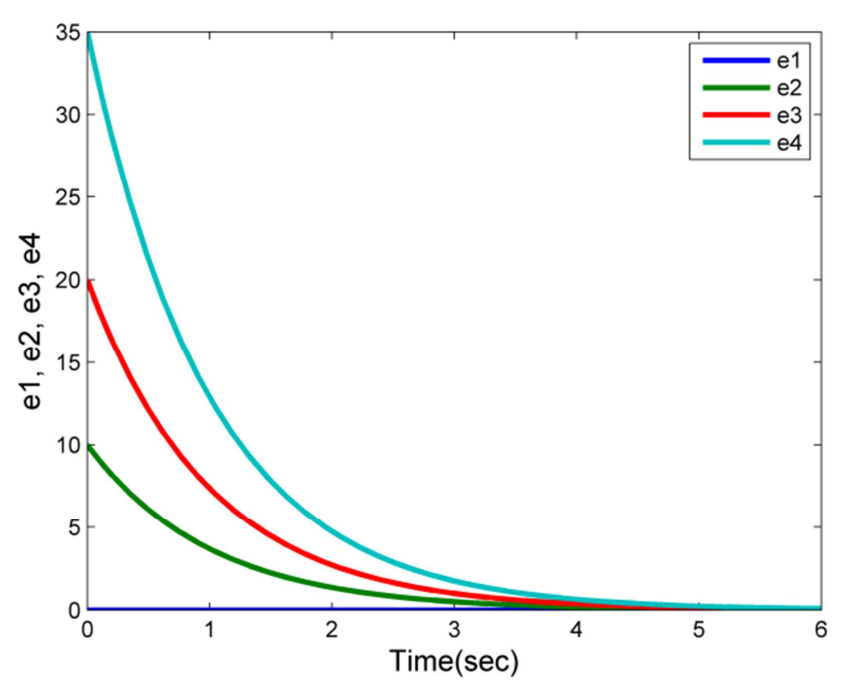

Fig. 6. The converges of system (16) with controllers (17) and update parameter (18).

\section{Hybrid Synchronization Between Modified Pan and Liu Systems}

Based on the adaptive control and Lyapunov second method the hybrid synchronization between two different 4D hyperchaotic systems, i.e. Modified Pan and Liu systems is consider in this section. Modified hyperchaotic Pan system is taken as drive (system 1) and hyperchaotic Liu system as response (system 3).

The hybrid synchronization error is defined by

$$
\left\{\begin{array}{l}
e_{1}=y_{1}-x_{1} \\
e_{2}=y_{2}+x_{2} \\
e_{3}=y_{3}-x_{3} \\
e_{4}=y_{4}+x_{4}
\end{array}\right.
$$

System (23) can be written in a succinct form as 


$$
e_{i}=\left\{\begin{array}{c}
y_{i}-x_{i}, \text { if } i \text { is odd } \\
y_{i}+x_{i}, \text { if } i \text { is even }
\end{array}\right.
$$

The error dynamics is easily obtained as

$$
\left\{\begin{array}{c}
\dot{e}_{1}=a\left(e_{2}-e_{1}\right)-2 a x_{2}+u_{1} \\
\dot{e}_{2}=r e_{1}+e_{4}+(r+c) x_{1}-k y_{1} y_{3}-x_{1} x_{3}+u_{2} \\
\dot{e}_{3}=-p e_{3}+(b-p) x_{3}+h y_{1}^{2}-x_{1} x_{2}+u_{3} \\
\dot{e}_{4}=-q e_{1}-d e_{2}-q x_{1}+d y_{2}+u_{4}
\end{array}\right.
$$

i.e. subtracting and adding the system (3) from the system (1) respectively.

Theorem 3. System (1) will hybrid synchronize with the system (3) with nine unknown parameters if adaptive control is chosen as

$$
\left\{\begin{array}{c}
u_{1}=(\hat{a}-1) e_{1}-\hat{a} e_{2}+2 \hat{a} x_{2} \\
u_{2}=-\hat{r} e_{1}-e_{2}-e_{4}+(\hat{r}+\hat{c}) x_{1}+\hat{k} y_{1} y_{3}+x_{1} x_{3} \\
u_{3}=(\hat{p}-1) e_{3}+(\hat{p}-\hat{b}) x_{3}-\hat{h} y_{1}^{2}+x_{1} x_{2} \\
u_{4}=\hat{q} e_{1}+\hat{d} e_{2}-e_{4}+\hat{q} x_{1}-\hat{d} y_{2}
\end{array}\right.
$$

with the parameter estimation update laws

$$
\left\{\begin{array}{c}
\dot{\hat{a}}=-e_{1}^{2}+e_{1} e_{2}-2 x_{2} e_{2}-\tilde{a} \\
\dot{\hat{b}}=x_{3} e_{3}-\tilde{b} \\
\dot{\hat{c}}=x_{1} e_{2}-\tilde{c} \\
\dot{\hat{d}}=-e_{2} e_{4}+y_{2} e_{4}-\tilde{d} \\
\dot{\hat{h}}=y_{1}^{2} e_{3}-\tilde{h} \\
\dot{\hat{k}}=-y_{1} y_{3} e_{2}-\tilde{k} \\
\dot{\hat{p}}=-e_{3}^{2}-x_{3} e_{3}-\tilde{p} \\
\dot{\hat{q}}=-e_{1} e_{4}-x_{1} e_{4}-\tilde{q} \\
\dot{\hat{r}}=e_{1} e_{2}+x_{1} e_{2}-\tilde{r}
\end{array}\right.
$$

Proof. Rewrite system (25) with control (26) and (8) as follows

$$
\left\{\begin{array}{c}
\dot{e}_{1}=\tilde{a}\left(e_{1}-e_{2}\right)-e_{1}+2 \tilde{a} x_{2} \\
\dot{e}_{2}=-\tilde{r} e_{1}-e_{2}+(\tilde{r}+\tilde{c}) x_{1}+\tilde{k} y_{1} y_{3} \\
\dot{e}_{3}=(\tilde{p}-1) e_{3}+(\tilde{p}-\tilde{b}) x_{3}-\tilde{h} y_{1}^{2} \\
\dot{e}_{4}=\tilde{q} e_{1}+\tilde{d} e_{2}-e_{4}+\tilde{q} x_{1}-\tilde{d} y_{2}
\end{array}\right.
$$

consider the Lyapunov function as

$$
\begin{gathered}
V=\frac{1}{2}\left(e_{1}^{2}+e_{2}^{2}+e_{3}^{2}+e_{4}^{2}+\tilde{a}^{2}+\tilde{b}^{2}+\tilde{c}^{2}+\tilde{d}^{2}+\tilde{h}^{2}+\tilde{k}^{2}+\tilde{p}^{2}+\tilde{q}^{2}+\tilde{r}^{2}\right) \\
\dot{V}=e_{1} \dot{e}_{1}+e_{2} \dot{e}_{2}+e_{3} \dot{e}_{3}+e_{4} \dot{e}_{4}+\tilde{a} \dot{\tilde{a}}+\tilde{b} \dot{\tilde{b}}+\tilde{c} \dot{\tilde{c}}+\tilde{d} \dot{\tilde{d}}+\tilde{h} \dot{\tilde{h}}+\tilde{k} \dot{\tilde{k}}+\tilde{p} \dot{\tilde{p}}+\tilde{q} \dot{\tilde{q}}+\tilde{r} \dot{\tilde{r}} \\
\dot{V}=e_{1}\left[\tilde{a}\left(e_{1}-e_{2}\right)-e_{1}+2 \tilde{a} x_{2}\right]+e_{2}\left[-\tilde{r} e_{1}-e_{2}+(\tilde{r}+\tilde{c}) x_{1}+\tilde{k} y_{1} y_{3}\right] \\
+e_{3}\left[(\tilde{p}-1) e_{3}+(\tilde{p}-\tilde{b}) x_{3}-\tilde{h} y_{1}^{2}\right]+e_{4}\left[\tilde{q} e_{1}+\tilde{d} e_{2}-e_{4}+\tilde{q} x_{1}-\tilde{d} y_{2}\right] \\
+\tilde{a}\left[-e_{1}^{2}+e_{1} e_{2}-2 x_{2} e_{2}-\tilde{a}\right]+\tilde{b}\left[x_{3} e_{3}-\tilde{b}\right]+\tilde{c}\left[x_{1} e_{2}-\tilde{c}\right]+\tilde{d}\left[-e_{2} e_{4}+y_{2} e_{4}-\tilde{d}\right] \\
+\tilde{h}\left[y_{1}^{2} e_{3}-\tilde{h}\right]+\tilde{k}\left[-y_{1} y_{3} e_{2}-\tilde{k}\right]+\tilde{p}\left[-e_{3}^{2}-x_{3} e_{3}-\tilde{p}\right]+\tilde{q}\left[-e_{1} e_{4}-x_{1} e_{4}-\tilde{q}\right]+\tilde{r}\left[e_{1} e_{2}+x_{1} e_{2}-\tilde{r}\right] \\
\dot{V}=-e^{T} e-\tilde{a}^{2}-\tilde{b}^{2}-\tilde{c}^{2}-\tilde{d}^{2}-\tilde{h}^{2}-\tilde{k}^{2}-\tilde{p}^{2}-\tilde{q}^{2}-\tilde{r}^{2}<0
\end{gathered}
$$

Therefore, hybrid synchronization between two hyperchaotic systems (1) and (3) with nine unknown parameters is achieved. The proof is now complete based on Lyapunov method. Fig. 7 and Fig. 8 show verify these results numerically.
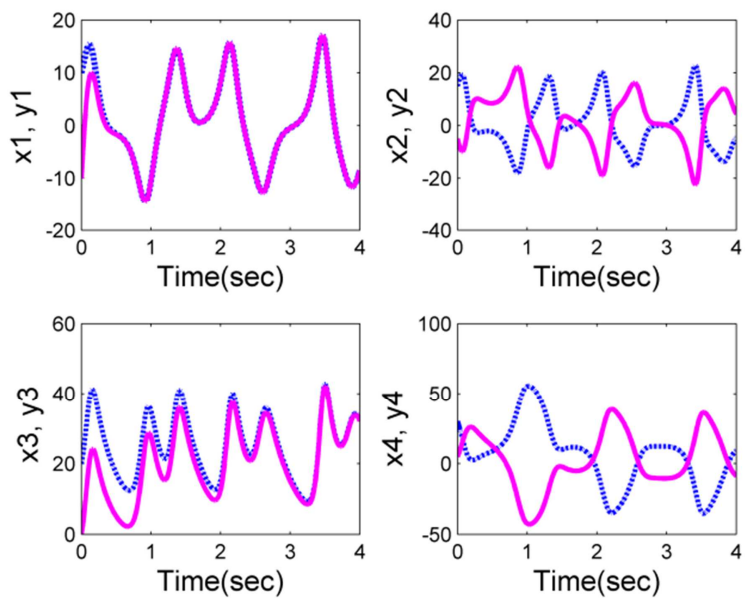

Fig. 7. Hybrid synchronization between systems (1) and (3) with adaptive control (26) and update parameter (27).

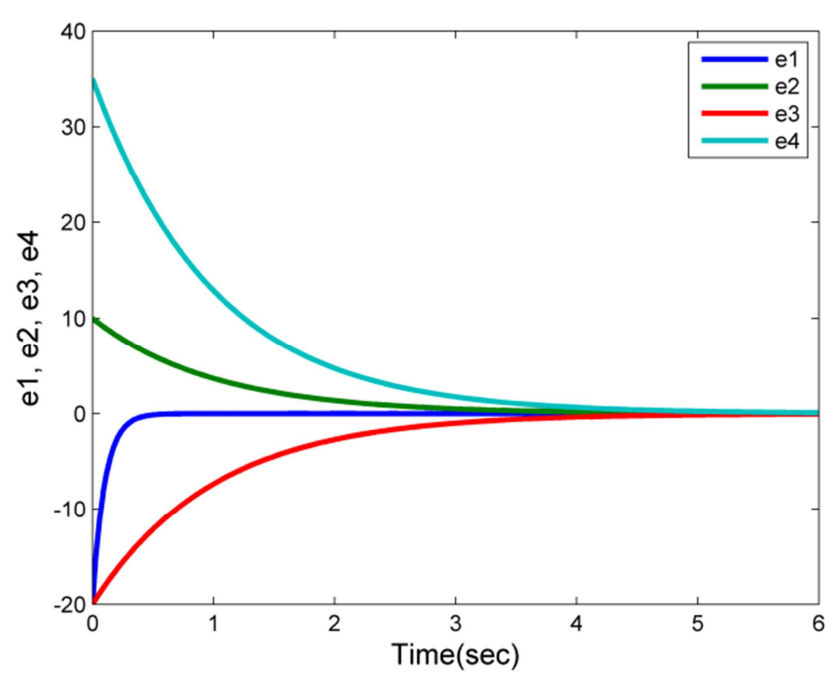

Fig. 8. The converges of system (25) with controllers (26) and update parameter (27).

\section{Conclusions}

In this paper deals with chaos synchronization between 
two non-identical hypechaotic with nine unknown parameters systems through nonlinear adaptive control technique. And succeed in achieving three important subjects which include complete synchronization, anti- synchronization and hybrid synchronization based on Lyapunov's second method. Numerical simulations are used to verify the effectiveness of the proposed control.

We believe that the results of this research work should be beneficial and could be employed to increase contribution to the scientific literature on the methods of chaos synchronization which may have applications in different fields of engineering including secure communication, hybrid image encryption, genetic networks.

\section{References}

[1] S. F. AL-Azzawi, Stability and bifurcation of pan chaotic system by using Routh-Hurwitz and Gardan methods, Appl. Math. Comput. 219, (2012) 1144-1152.

[2] M. M. Aziz., S. F. AL-Azzawi, Chaos control and synchronization of a novel 5-D hyperchaotic Lorenz system via nonlinear control, Int. J. Mode. Phys. Appli., 2, (2015) $110-115$.

[3] G. Cai, Z. Tan, Chaos synchronize of a new chaotic system via nonlinear control, J. Uncertain Systems, 1 (2007) 235-240.

[4] HK. Chen, Global Chaos synchronization of new chaotic systems via nonlinear control, Chaos, Solitons and Fractals, 23 (2005) 1245-1251.

[5] F. Yu, C. H. Wang, Y. Hu, J. W. Yin, Anti-synchronization of a novel hyperchaotic system with Parameter mismatch and external disturbances, journal of physics, Pramana. 79 (2012) 81-94.

[6] F. Q. Dou, J. A. Sun, W. S. Duan, K. P. Lü, Controlling hyperchaos in the new hyperchaotic system, Commun. Nonlinear Sci. Numer., 14 (2009) 552-559.

[7] E. M. Elabbasy, H. N. Agiza, M. M. El-Dessoky, Global Chaos synchronization for four - scroll attractor by nonlinear control. Scientific Research and Essay, 1 (2006) 65-71.
[8] Q. Jia, Hyperchaos synchronization between two different hyperchaotic systems, Journal of Information and Computing. Science, 3 (2008) 73-80.

[9] A. Khan, P. Singh, Chaos synchronization in Lorenz system, Appl. Math., 6 (2015), 1864-1872.

[10] D. Lu, A. Wang, X. Tian, Control and synchronization of a new hyperchaotic system with unknown parameters, International Journal of Nonlinear Science. 6 (2008) 224-229.

[11] L. Pan, W. Zhou, L. Zhou, K. Sun, Chaos synchronization between two different fractional-order hyperchaotic systems, J, Commun. Nonlinear Sci. Numer. Simulat., 16 (2011), 26282640 .

[12] J. H. Park, Chaos synchronization of a chaotic system via nonlinear control, Chaos Solitons Fractals 25 (2005) 579-584.

[13] F. Q. Dou, J. A. Sun, W. S. Duan, Anti-synchronization in different hyperchaotic systems, Commun. Theor. Phys., 50 (2008) 907-912.

[14] P. P. Singh, J. P. Singh, B. K. Roy, Synchronization and antisynchronization of Lü and Bhalekar-Gejji chaotic systems using nonlinear active control. Chaos, Solitons and Fractals, 69 (2014) 31-39.

[15] M. Srivastava, S. P. Ansari, S. K. Agrawal, S. Das, A. Y. T. Leung, Anti- synchronization between identical and nonidentical fractional-order chaotic systems using active control method, Nonlinear Dyn. 76 (2014) 905-914.

[16] K. S. Sudheer, M. Sabir, Hybrid synchronization of hyperchaotic Lü system, Pramana J. Phys.73 (2009) 781-786.

[17] G. Xu, S. Chen, Hybrid synchronization of a Chen hyperchaotic system with two simple linear feedback controllers Appl. Math., 4 (2013), 13-17.

[18] M. M. Aziz., S. F. AL-Azzawi, Control and synchronization with known and unknown parameters, Appl. Math., 7 (2016), 292-303.

[19] FQ. Wang, CX. Liu, Hyperchaos evolved from the Liu chaotic system. Chin Phys 15 (2006), 963-970. 